\title{
Les connaissances professionnelles des enseignants et leur évolution à travers une analyse de l'activité. Une étude de cas en physique au collège
}

Teachers' professional knowledge and their evolution through an analysis of the activity. A case study at middle school

\section{Alain Jameau}

\section{OpenEdition \\ Journals}

\section{Édition électronique}

URL : https://journals.openedition.org/educationdidactique/2140

DOI : $10.4000 /$ educationdidactique. 2140

ISBN : 978-2-7535-4191-7

ISSN : 2111-4838

Éditeur

Presses universitaires de Rennes

\section{Édition imprimée}

Date de publication : 20 mai 2015

Pagination : 9-31

ISBN : 978-2-7535-4146-7

ISSN : 1956-3485

\section{Référence électronique}

Alain Jameau, « Les connaissances professionnelles des enseignants et leur évolution à travers une analyse de l'activité. Une étude de cas en physique au collège », Éducation et didactique [En ligne], 9-1 mai 2015, mis en ligne le 20 mai 2017, consulté le 23 août 2022. URL : http://

journals.openedition.org/educationdidactique/2140; DOI : https://doi.org/10.4000/ educationdidactique. 2140 


\title{
LES CONNAISSANCES PROFESSIONNELLES DES ENSEIGNANTS ET LEUR ÉVOLUTION À TRAVERS UNE ANALYSE DE LACTIVITÉ \\ UNE ÉTUDE DE CAS EN PHYSIQUE AU COLLÈGE
}

Alain Jameau

CREAD, Université de Bretagne Occidentale, ESPE de Bretagne

\begin{abstract}
Cet article porte sur le thème des connaissances professionnelles mobilisées par les enseignants de physique-chimie. Nous nous plaçons dans le contexte de l'enseignement de la mécanique en classe de troisième. Notre approche théorique articule la didactique des sciences et la didactique professionnelle. Nous avons élaboré une méthodologie spécifique dont l'un des principes est d'analyser l'écart entre la préparation des enseignements et leur mise en œuvre. Nous articulons le modèle des PCK et le concept de schème pour identifier les connaissances professionnelles. Nous montrons qu'il y a une relation entre ces connaissances et la régulation rétroactive de l'activité qui permet à l'enseignant d'ajuster sa préparation. Nous discutons des intérêts du croisement des deux didactiques pour décrire l'activité des enseignants et les apprentissages des élèves, notamment au cours d'un processus de modélisation en sciences expérimentales.
\end{abstract}

Mots-clés : connaissances pédagogiques liées au contenu, PCK, schème, didactique des sciences, didactique professionnelle, boucles de régulation.

Teachers' professional knowledge and their evolution through an analysis of the activity.

A case study at middle school

This paper focuses on the theme of professional knowledge mobilized by physics' teachers. Our study takes place in the context of the teaching of Mechanics in grade 9 in France. Our theoretical approach refers to science education and professional didactics. We have developed a specific methodology and one of its principles is to analyze the gap between the preparation of lessons and their implementation. We articulate the model of PCK and the concept of scheme to identify professional knowledge. We show that there is a relationship between knowledge and retroactive control that allows the teacher to adjust in/her preparation. We discuss the interests of the intersection of the two didactics to describe the activity of teachers and students learning, especially in a process of modeling in experimental sciences.

Keywords: pedagogical content knowledge, PCK, scheme, science education, professional didactic, regulation loop. 
Cette étude examine les connaissances mobilisées par des professeurs dans leur enseignement de physique-chimie au collège, en France. Elle nécessite de prendre en compte l'activité de l'enseignant dans l'interactivité professeur-élève car nous postulons que certains types de connaissances sont construites pendant cette interactivité. Pour identifier les connaissances professionnelles, nous analysons l'organisation de l'activité des professeurs ainsi que les tâches prescrites pour les élèves. Nous étudions donc, d'un côté, le savoir et sa transmission et, de l'autre côté, l'activité des enseignants en contexte de travail. Le premier objet d'étude est généralement analysé par la didactique disciplinaire alors que le second est examiné par la didactique professionnelle en considérant, en particulier, que activité et apprentissage sont indissociables (Pastré, 2008). En ce qui nous concerne, pour étudier les tâches prescrites aux élèves, nous nous appuyons sur les travaux en didactique des sciences et, plus particulièrement, sur ceux de Martinand (1996) concernant la modélisation en sciences.

Nous étudions le processus qui permet à l'enseignant d'adapter sa préparation ${ }^{1}$ à un public et comment celle-ci évolue en relation avec l'activité dans la classe. Nous nous intéressons spécifiquement à l'écart entre ce qui est prévu par le professeur et ce qui est réalisé en classe. Nous devons donc considérer ce qui se passe hors de la classe et dans la classe. Selon Bécu-Robinault (2007), une part non négligeable des décisions sur l'enseignement est prise pendant le travail hors classe et notamment lors de la préparation. Nous y accédons à partir des traces écrites produites par le professeur, de son journal de bord et des entretiens.

Nous exposons en première partie les cadres théoriques sur lesquels nous nous appuyons pour cette recherche et notre questionnement dans la seconde partie. Dans la troisième partie, nous présentons notre méthodologie construite selon trois principes : un suivi simultané de deux enseignants englobant leur activité hors classe ; une comparaison de ce qui est prévu et de ce qui est réalisé en classe; la confrontation du professeur à son action par le biais de vidéos et d'autoconfrontations. Dans la quatrième partie, nous présentons un extrait d'étude de cas en physique-chimie au collège dont nous discutons les résultats dans la cinquième partie. Enfin, nous donnons des éléments de conclusion en interrogeant en particulier certains éléments de notre cadre théorique et nous évoquons quelques perspectives.

\section{ProblématiQue ET CADRE THÉORIQUe}

L'étude des connaissances professionnelles des enseignants a déjà été abordée dans de nombreuses recherches en éducation au niveau international, aussi bien d'un point de vue général que du côté des professeurs de sciences. Elles réfèrent souvent au concept de PCK (Shulman, 1986, 1987) qui peut être traduit par connaissances pédagogiques liées au contenu. Il s'agit d'une connaissance spécifique pour enseigner qui est nourrie partiellement par la connaissance du contenu (Sensevy \& Amade-Escot, 2007). La plupart des études internationales, comme le montre Abell (2007), ont cherché à identifier les connaissances des enseignants à partir de ce qu'ils disent sur leurs connaissances et de leur pratique. En France, les études sont encore peu nombreuses. Certaines mettent en œuvre des méthodologies qui permettent de repérer une PCK à partir de l'action du professeur (Bécu-Robinault, 2007 ; Kermen \& Méheut, 2008 ; Cross, 2010 ; Jameau, 2012). Quelques-unes soulignent la nécessité de se placer à un grain plus petit pour mieux conceptualiser une PCK. Par exemple, Cross (2010) étudie les Teacher Pedagogical Construction (TPC) qui sont une collection d'unités plus petites que les PCK tandis que Morge (2008) parle des Local Pedagogical Content Knowledge (LPCK) qui sont des unités dépendant du contenu enseigné et qui ne peuvent pas faire l'objet d'un transfert à d'autres séances. Dans tous les cas, ce modèle originel est très peu discuté car, soit il est redéfini à chaque recherche, soit un autre cadre théorique est proposé (Abell, 2007).

Notre étude nous conduit à explorer différents cadres théoriques que nous présentons maintenant. Tout d'abord, nous donnons quelques éléments à propos de l'articulation entre la didactique des disciplines et la didactique professionnelle, pour comprendre comment les connaissances sont construites par les professeurs. Puis, nous exposons nos références à la didactique professionnelle pour analyser l'organisation de l'activité des enseignants et les mécanismes de régulations qui accompagnent l'activité. Enfin, nous examinons les travaux de recherche en didactique des sciences concernant la modélisation et nous revenons sur le concept de PCK avant de présenter le modèle de Magnusson, Krajcik, et Borko, (1999) que nous utilisons dans notre analyse. 
À propos de l'articulation entre la didactique des disciplines et la didactique professionnelle

Pour Pastré (2011), ces deux didactiques se différencient notamment au niveau de la différence d'objet d'étude, entre la connaissance et l'activité. Cette différence peut se retrouver dans l'importance qui est accordée, lors d'une analyse, à la mise en œuvre d'une connaissance et à la mise en œuvre d'une activité. Dans le premier cas, l'essentiel de la focalisation va porter sur les conditions d'accession d'une connaissance; dans le second cas, l'essentiel de l'analyse portera sur les modalités de déploiement de l'activité. Pour autant, dans les deux cas, il y a à la fois des connaissances en jeu et de l'activité mais avec des buts différents : produire une action efficace ou construire des connaissances. Cette différence de but aboutit « à un renversement de la relation de subordination entre connaissances et actions $»$ (Pastré, 2011, p. 19). Pastré montre que cela correspond à un passage d'un registre épistémique à un registre pragmatique que Munoz (2006) décrit comme un changement d'objectifs. Pour Vergnaud (2008), les caractéristiques d'un métier sont différentes de celles d'une discipline, ce qui produit des problématiques tout aussi différentes. De son côté, Rabardel (2007) souligne que la didactique professionnelle se situe dans une autre perspective épistémologique suivant deux dimensions principales : le pouvoir de faire du sujet, son pouvoir d'agir et sa capacité à construire des ressources, à se les approprier pour assurer ce pouvoir de faire.

Un autre point de divergence apparaît dans «l'opposition » entre le singulier de la didactique professionnelle et le pluriel des didactiques disciplinaires (Pastré, 2011). Pour autant, ce problème d'articulation entre le singulier et le pluriel est un des éléments qui a permis aux deux didactiques de se rapprocher. En effet, les concepts de situation didactique, de transposition, de contrat, de schème et de champ conceptuel trouvent un écho en didactique professionnelle (Pastré, Vergnaud, \& Mayen, 2006). Pastré (2008) constate ainsi qu'il y a de l'activité dans l'apprentissage d'une connaissance et qu'il y a mobilisation de connaissances dans l'apprentissage d'une activité ce qui constitue, pour lui, un autre élément de rapprochement.

\section{L'organisation de l'activité et les boucles de régulations}

Nous faisons référence, à propos de l'organisation de l'activité, au concept de schème suivant le développement théorique de Vergnaud (1990) s'appuyant lui-même sur les travaux de Piaget (1974). Nous en donnons une définition avec ses composantes. Ensuite, nous présentons le modèle de régulation dans une analyse de l'activité.

\section{Le schème comme unité élémentaire d'activité}

Vergnaud reprend la notion centrale de schème en l'articulant à la notion de situation. Un schème est " une organisation invariante de la conduite pour une classe de situations données " (Vergnaud, 2001, p. 112). Le concept de classe de situations signifie des événements porteurs d'une même structure conceptuelle et qui vont convoquer chez ceux qui y sont confrontés des schèmes d'actions spécifiques nécessaires à leur adaptation (Vinatier, 2009). Vergnaud souligne que les situations vécues sont à la fois source et critère de connaissances. C'est en situation qu'on apprend. Autrement dit, une connaissance qui n'est pas opératoire n'est pas une connaissance. Par conséquent, le lien entre les classes de situations et les concepts qui s'y trouvent à l'œuvre est fort.

Vergnaud donne des définitions complémentaires du schème. La première définition est d'ordre fonctionnel. Il distingue l'activité et son organisation car «le schème n'est pas un stéréotype : ce qui est invariant, c'est l'organisation, non pas l'activité et la conduite " (Vergnaud \& Récopé, 2000, p. 45). L'activité est contextualisée, variable et adaptée aux circonstances (Pastré, 2011). La seconde définition est d'ordre analytique. Elle exprime l'idée que le schème est ce qui engendre l'activité au fur et à mesure de son déroulement.

Selon Vergnaud (1996), le schème repose sur les quatre éléments suivant :

Le but, les sous-buts, les anticipations. Il représente dans le schème l'intention, le désir, le besoin, la motivation, l'attente. Cet élément précède et accompagne l'action. Le but se décline en sous-buts et anticipations de la même manière que les schèmes se décomposent et se recomposent hiérarchiquement ; Les règles d'action, de prise d'information et de contrôle. C'est la partie générative du schème. 
Ces règles d'action engendrent toute l'activité aussi bien les prises d'information et les contrôles que les actions matérielles. Elles conduisent au résultat recherché. Le prélèvement et la sélection de l'information pertinente se font par les invariants opératoires pour en inférer « des conséquences utiles pour l'action, le contrôle et la prise d'information subséquente » (Vergnaud \& Récopé, 2000, p. 47) ; Les concepts-en-acte et des théorèmes-en-acte. Ils constituent la partie épistémique. Il y a des propositions (théorèmes-en-acte) qui sont susceptibles d'être vraies ou fausses et des objets ou des prédicats (conceptsen-acte) qui ne sont ni vrai ni faux mais seulement pertinents ou non pertinents pour la prise d'information nécessaire à l'action. Vergnaud précise que la relation entre concepts et théorèmes est dialectique ; Les inférences. Elles sont présentes dans toutes les activités en situation car une activité n'est jamais automatique.

C'est l'identification de ces quatre composantes qui permet de comprendre la structure de l'activité.

\section{Les boucles de régulation}

Selon Leontiev (1972), une activité se réalise par des actions ; l'action répond à un but. Dans une situation de travail, ce but fait partie de la tâche que Leontiev définit comme « un but donné dans des conditions déterminées » (p. 96). Leplat (2004) précise la nécessité de distinguer tâche et activité. La tâche c'est "le but à atteindre et les conditions dans lesquelles il doit être atteint » alors que l'activité c'est «ce qui est mis en œuvre par le sujet pour exécuter la tâche » (ibid., p. 14).

Leplat (2006) signale que la notion de régulation revient souvent dans les textes consacrés à l'étude de l'activité en situation de travail. Et, comme le souligne Coulet (2011): "Il est, en effet, difficile de rendre compte de l'activité sans insister sur les mécanismes de régulation qui l'accompagnent » (p. 15). Par conséquent, Leplat propose une définition et un modèle qui lui permettent de montrer « comment un modèle de régulation peut mettre en lumière certains aspects de l'activité » (Leplat, 2006, p. 5). Il mentionne quelques principaux types de régulations qu'il classe comme des régulations rétroactives et proactives : la première est fondée sur les résultats, la seconde sur l'antici- pation. Coulet (2010) ajoute que «les régulations proactives ont pour fonction l'ajustement d'un schème au regard de la spécificité de la situation à travers la variabilité des actions portées, tandis que les régulations rétroactives montrent la réorganisation de l'activité en conséquence des retours sur l'action (feedback) pris en compte par le sujet» (p. 5).

De son côté, Pastré (1999) montre, dans le cadre de sa recherche sur l'apprentissage de la conduite de centrales nucléaires sur simulateurs, qu'il existe deux types de stratégies, qualifiées toutes les deux de « rétroactives et partielles ». Le novice ne parvient pas à avoir une représentation d'ensemble du fonctionnement; sa stratégie dite à boucle courte est de type procédural. Le résultat de la formation sur simulateur permet à Pastré de qualifier la seconde stratégie de boucle longue; elle est de type analytique. Autrement dit, le novice modifie son activité de proche en proche, suivant un mode que l'on pourrait qualifier d'essai/erreur, où à chaque défaut correspond une règle d'action. La boucle courte est une régulation de type " coordination agie » (Piaget, 1974), essentiellement axée vers la réussite. En ce qui concerne la boucle longue, l'opérateur met en œuvre une forme de " coordination conceptuelle » (Piaget, 1974), à travers une approche globale. Il apparaît, de plus, dans des travaux sur la résolution de problèmes, une autre forme de régulation rétroactive de l'activité : celle qui réoriente le sujet vers d'autres formes d'activités, vers d'autres schèmes, qui seraient plus adaptés aux propriétés de la situation et de la tâche. Pour Coulet (2010), il s'agit de régulations de type " changement de schème».

Cette présentation des concepts de schème et de régulation apporte des outils conceptuels pour nous aider à analyser l'activité des enseignants, son organisation, et les processus de régulation qui l'accompagnent. En effet, l'articulation opérée par Vergnaud, entre la notion de schème et celle de situation, permet de montrer que toute activité est organisée. C'est dans les invariants opératoires que vont se retrouver les connaissances des enseignants. Nous faisons la relation entre des propositions, les concepts et les connaissances des enseignants.

Nous examinons maintenant les travaux de recherche concernant la modélisation dans l'enseignement des sciences afin d'étudier la tâche prescrite aux élèves en lien avec l'activité des enseignants, dans le cas d'un enseignement de physique. 
La modélisation dans l'enseignement des sciences

D'après Boilevin (2013), « la place des modèles dans la pratique scientifique est essentielle si l'on considère que la science constitue un système de représentation du monde qui nous entoure » (p. 63). Certaines recherches en didactique s'intéressent à sa prise en compte dans l'enseignement. Ainsi, pour Martinand (2000), l'enseignement des sciences, trop dogmatique, présente les modèles comme des évidences non questionnées non rattachées à des problèmes. Les questions d'enseignement et d'apprentissage de la modélisation se posent en termes de construction, d'adaptation et d'utilisation des modèles scientifiques. Ceux-ci doivent conserver au cours des manipulations leurs trois caractéristiques essentielles : ils sont hypothétiques, modifiables et ils sont pertinents pour certains problèmes dans certains contextes.

Fondé sur les travaux de Walliser (1977), le schéma de la modélisation (figure 1) proposé par Martinand permet de décrire la tâche de l'élève durant l'apprentissage. Ce schéma distingue le registre du référent empirique et le registre des modèles construits sur ces référents, selon des exigences qui n'ont pas de solution dans le premier registre (Martinand, 2000). Le registre du référent empirique n'est pas constitué uniquement d'objets ou de phénomènes, ce qui pourrait être appelé le réel, mais aussi de connaissances phénoménographiques, phénoménotechniques et phénoménologiques qui leur sont associées. Il s'agit des descriptions, des règles d'actions, des savoirs disponibles, qui sont issus de processus antérieurs et qui sont inconsciemment projetés sur la réalité, ce qui justifie qu'ils aient un statut empirique. Bisault (2011) ajoute que ce qui est référent, à un moment donné, est une description qui remplace la réalité qu'on ne connaît pas. De la même manière, un modèle scientifique peut se substituer à une description antérieure et être incorporé comme phénoménologie dans un nouveau registre empirique (Larcher, 2003).

Pour Martinand, il y a une responsabilité didactique dans le choix et la définition du référent empirique car les objets et les phénomènes ne sont pas « donnés » mais ils sont le résultat d'une lecture de la « réalité ». Cependant, cette lecture n'est pas forcément la même pour les élèves et pour des adultes instruits (Martinand, 2000).

Le registre des modèles comprend des composantes " sémantiques », " syntaxiques » et " pragmatiques » que Sanchez (2008) décrit comme une sémiographie " commode " pour représenter les éléments du modèle. Ce registre décrit le modèle scientifique mobilisé qui permet d'interpréter et de représenter le réel.

Martinand précise que ce schéma permet aussi de distinguer une description première devant donner lieu à une construction consensuelle (phénoménographie), et une description seconde où le modèle se projette sur le référent (phénoménologie).

Figure 1. Schéma sur la modélisation (Martinand, 1996)

\section{Modèles}

\section{Référent}

empirique

\section{Sémantique, pragmatique, syntaxe}

Représentations symboliques

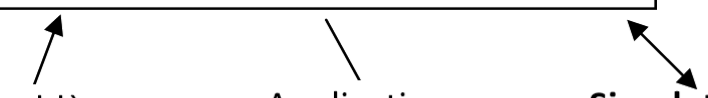

Tâche ou problème

"Application »

impliquant modélisation

Phénoménographie

du modèle

Simulation avec

Phénoménotechnique 
Ces références théoriques à la didactique des sciences nous permettent d'étudier la tâche prescrite aux élèves au cours d'une modélisation et dans l'application du modèle. Nous faisons la relation avec le but du professeur dans l'activité et les changements de but grâce aux concepts de schème et de régulation. Dès lors, nous pouvons regarder la manière dont les élèves s'approprient les savoirs en jeu. Il ne nous reste plus qu'à présenter le concept de PCK et le modèle de Magnusson et al. (1999) qui nous permettent d'analyser les connaissances des enseignants afin de montrer leur impact au niveau de leur activité et de sa conduite.

\section{Pedagogical Content Knowledge}

Le modèle originel proposé par Shulman, en 1986, vise la compréhension des connaissances spécifiques en jeu, dans l'enseignement d'un savoir en lien avec un sujet, afin de distinguer un enseignant d'un spécialiste de ce sujet. Il définit les connaissances pédagogiques liées au contenu, Pedagogical Content Knowledge (PCK). Elles expriment la combinaison (l'amalgame) du contenu et de la pédagogie. La description la plus complète se trouve dans l'étude de Grossman (1990), qui définit les quatre grands domaines de connaissance de l'enseignant: les connaissances pédagogiques générales (PK), les connaissances disciplinaires (SMK), les connaissances pédagogiques liées au contenu disciplinaire (PCK), et les connaissances du contexte (KofC). De leur côté, Magnusson et al. (1999) définissent un modèle des PCK suivant cinq composantes que nous retenons pour notre étude (figure 2) :

Connaissances sur l'orientation pour l'enseignement des sciences : elles se réfèrent aux connaissances des enseignants à propos des buts et des objectifs pour enseigner les sciences à un niveau d'étude particulier ; Connaissances des programmes de sciences : elles concernent la connaissance des buts et objectifs (attentes nationales) et la connaissance spécifique du programme et du matériel pédagogique ; Connaissances sur l'évaluation : ce qu'il faut évaluer et comment l'évaluer ; Connaissances des stratégies d'enseignement : elles incluent des stratégies spécifiques à l'enseignement de certains sujets. Par exemple, sur un cycle d'ap-

Figure 2. Components of pedagogical content knowledge for science teaching (Magnusson et al., 1999)

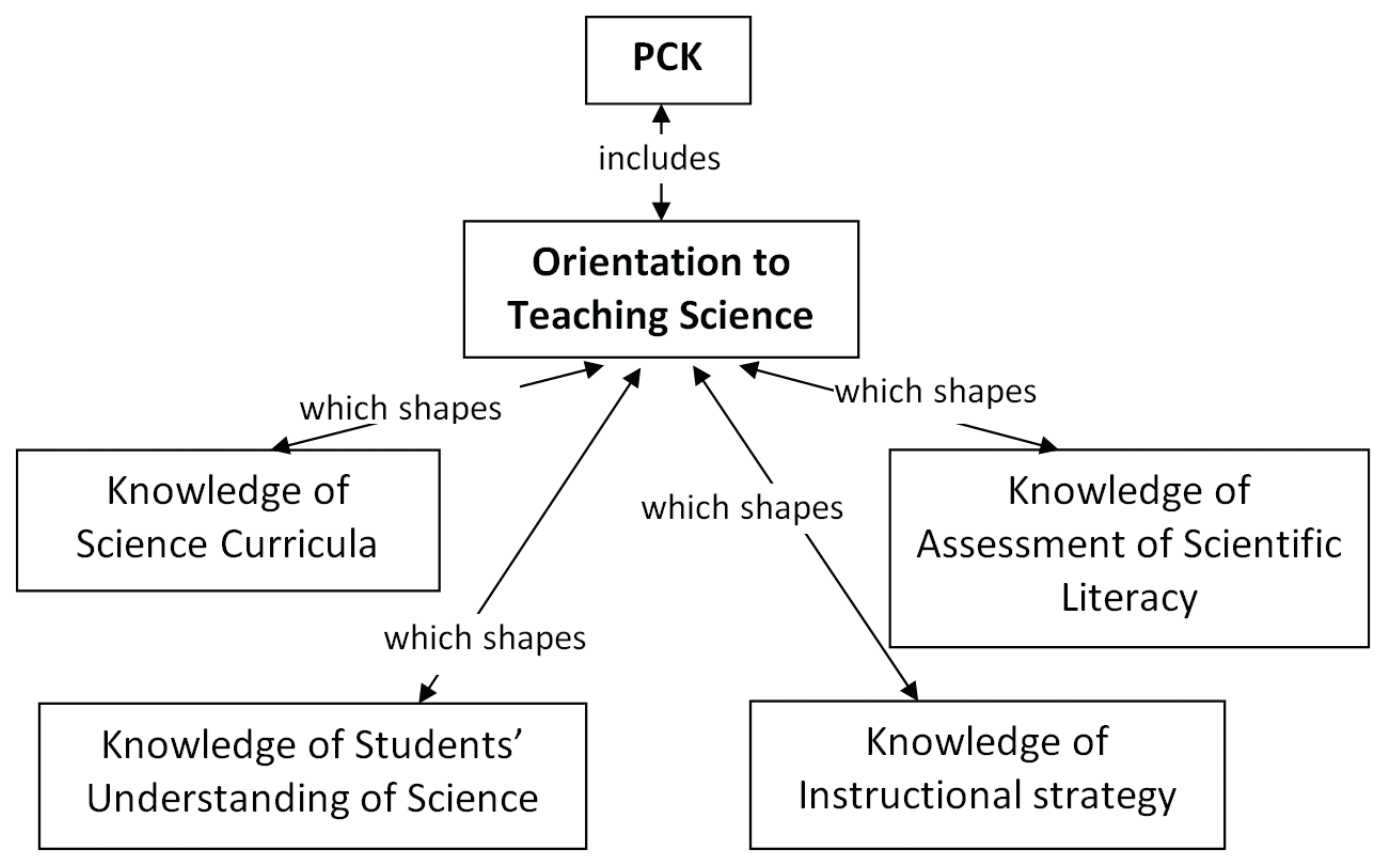


prentissages, l'utilisation d'analogies, de démonstrations ou d'expériences ; Connaissances sur les élèves : elles portent sur la connaissance que les enseignants ont des apprentissages des élèves en sciences : les exigences de l'apprentissage de certaines notions, les parties que les élèves trouvent difficiles, les approches de l'apprentissage des sciences, et les conceptions alternatives.

Ces cinq composantes des PCK sont, elles-mêmes, divisées en sous-catégories. La composante « orientations pour l'enseignement $»$ chapeaute les autres. Cependant, son inclusion dans le modèle des PCK est questionnée pour deux raisons (Abell, 2007). Premièrement, une orientation est théorisée comme une vue générale de l'enseignement des sciences, et non pas comme des connaissances spécifiques d'un sujet.

Deuxièmement, ces vues générales de l'enseignement des sciences et des apprentissages sont souvent étudiées en tant qu'interaction entre les connaissances, les croyances et les valeurs, et non pas strictement comme une structure de connaissances.

Dans notre étude, ce modèle de Shulman (1986, 1987) modifié par Grossman (1990) et Magnusson et al., (1999) est précieux pour reconstruire les connaissances en jeu dans la pratique de l'enseignant. Il permet de distinguer les connaissances qui se situent au niveau enseignant, celles qu'il mobilise en lien avec un contenu à enseigner, des connaissances au niveau élève qui sont les connaissances spécifiques à l'enseignement de ce contenu. Mais ce travail de reconstruction de connaissances à partir de l'action de l'enseignant dans la classe est difficile car, selon Vergnaud (1996), c'est la forme opératoire de la connaissance qui est utilisée dans l'action. Or, elle est en décalage avec la forme prédicative, ce qui signifie qu'il existe des connaissances implicites, qui sont difficilement identifiables.

\section{Questions de recherche}

Les cadres théoriques que nous venons de présenter nous permettent de préciser nos questions de recherche. Afin d'étudier l'évolution des connaissances professionnelles en jeu, nous devons articuler l'action des enseignants avec leurs connaissances. Pour cela, nous examinons l'organisation de leur activité, représentée par le concept de schème.
Cette organisation s'appuie sur le présupposé que les connaissances professionnelles se trouvent dans les invariants opératoires. Nous mobilisons le concept de PCK comme cadre d'analyse des connaissances des enseignants en nous référant au modèle de Magnusson et al., (1999) pour caractériser les types de connaissances professionnelles en jeu. Notre problématique s'articule autour de deux questions principales: Quels types de connaissances professionnelles sont mobilisés par les enseignants? Comment évoluent les connaissances au cours de l'activité du professeur?

Le concept de boucle de régulation rétroactive de l'activité permet d'analyser les conséquences de l'écart entre le prévu et le réalisé. Par conséquent, la seconde question principale peut se décliner en sous-questions de la manière suivante : Comment se construisent les nouvelles connaissances? De quel(s) type(s) sont-elles ? Comment participent-elles à l'organisation de l'activité et à sa conduite?

Pour répondre à ces questions, nous avons construit une méthodologie propre à cette recherche que nous présentons maintenant.

\section{MÉTHOdOLOGIE}

Nous explicitons, dans ce paragraphe, les outils de recueil et de traitement des données par rapport au contexte de notre étude.

\section{Contexte de l'étude}

Nous avons mis en œuvre cette méthodologie pendant deux années consécutives, pour le suivi de deux enseignants expérimentés de collège, spécialistes d'un enseignement disciplinaire : la physiquechimie. Nous les avons appelés Henri et Florence. L'étude de cas que nous présentons ici se réfère à un enseignement en mécanique, dans les programmes de la classe de $3^{\mathrm{e}}$ en vigueur à partir de 2008. Ce choix permet d'éviter, d'un côté, les enseignants débutants et les problèmes inhérents au début de carrière qui pourraient occulter les objets de notre étude, et de l'autre, les enseignants experts qui enseignent au même niveau depuis des années, chez qui nous pourrions observer des préparations pouvant être succinctes, et des routines installées qui rendraient moins visibles les évolutions qui nous intéressent. 
Le thème choisi concerne les notions de «poids et masse »; il est traité par chacun des enseignants en trois séances d'une heure chacune. Les progressions ont été coordonnées de manière à ce que ce chapitre soit enseigné dans la même période de l'année afin de permettre à chacun de discuter de situations d'enseignement récemment vécues.

\section{Outils de recueil de données}

Le corpus que nous recueillons comporte des enregistrements audio et vidéo de séquences de classe et des entretiens avec chacun des enseignants, ainsi que des données issues d'un journal de bord renseigné par les deux professeurs sur toute la durée de l'étude. Ce dernier nous donne une trace de leur préparation de classe et de leur analyse de la séance précédente. Il permet d'approcher le travail hors classe des enseignants. C'est un outil essentiel de la méthodologie d'investigation réflexive dans le sens où il incite à la réflexivité sur les activités (Power, 2008 ; Gueudet \& Trouche, 2010).

La durée totale d'enregistrement vidéo est d'environ 38 heures. Nous avions deux caméras à disposition : la première était fixe au fond de la classe centrée sur le tableau et la seconde était mobile prenant les interactions entre le professeur et les élèves. L'enseignant était équipé d'un micro-cravate et deux micros «d'ambiance » étaient placés dans la classe. L'enregistrement vidéo était ensuite numérisé.

Nous menons différents types d'entretiens avec les enseignants : un entretien en début et en fin de séquence ainsi que des entretiens de type autoconfrontation (Clot \& Faïta, 2000 ; Clot, Faita, Fernandez, \& Scheller, 2001). Le thème étudié lors de la séquence a été décidé au préalable conjointement par les enseignants et le chercheur. Il sert de base à deux entretiens dans lesquels les professeurs font une auto-analyse de leur action, à partir des enregistrements vidéo des séances, selon des modalités proches de l'autoconfrontation simple et croisée. L'auto-analyse est envisagée ici comme méthode de recueil de données empiriques et d'analyse de protocoles verbaux en relation avec l'action. L'auto-analyse simple consiste en un entretien entre le chercheur et chaque enseignant. Il leur est demandé de décrire puis d'analyser leurs actions, en verbalisant ce qu'ils faisaient, pensaient ou prenaient en compte pour agir, et en évitant les interprétations ou généralités.
L'auto-analyse croisée met en scène deux enseignants et le chercheur dans une analyse commune du même enregistrement vidéo. Il s'agit d'analyser les incidents critiques relevés au cours de l'auto-analyse simple. Nous avons donc retenu de suivre deux enseignants simultanément pour pouvoir organiser ces entretiens.

\section{Outils de traitements des données}

Notre analyse se situe parfois à un grain très fin, au niveau de l'énoncé, pour nous permettre de percevoir les ajustements effectués par les enseignants dans l'action de la classe ce qui nous a conduit à procéder selon la méthodologie de l'étude de cas, comportant deux niveaux d'analyse.

Au premier niveau, nous élaborons un synopsis (Sensevy \& Mercier, 2007) des séances à partir d'une première analyse vidéo. D'un point de vue méthodologique, le synopsis de séance correspond à une réduction du corpus permettant une vision de l'ensemble de la séance étudiée. Afin de préparer l'entretien en auto-analyse simple, nous fournissons à chacun des enseignants les vidéos de classe de leur propre pratique avec les synopsis. Puis, nous leur demandons de relever toutes les situations qu'ils souhaiteraient discuter avec leur binôme en notant, en particulier, tous les imprévus survenus en classe. Nous appelons un imprévu une réponse d'élève, une attitude, une difficulté inattendue dans une activité, etc., tout ce qui n'a pas été anticipé par le professeur. Au cours de cet entretien, les imprévus identifiés par l'enseignant sont croisés avec ceux relevés par le chercheur, puis discutés. Ceux qui sont jugés pertinents, au regard de notre étude, sont définis comme incidents critiques (Flanagan, 1954). Nous établissons une liste des incidents critiques à discuter lors de l'entretien croisé et nous donnons à chaque enseignant les vidéos des cours dispensés par l'autre membre du binôme ainsi que les synopsis des séances. Ils doivent en faire une première analyse et relever des objets à discuter avec leur binôme. Cela peut concerner la progression, la planification, une situation, etc. Ce corpus sera la base de l'entretien en auto-analyse croisée. Au second niveau, nous élaborons les transcriptions des situations qui ont été discutées dans les entretiens. Elles sont relatives notamment aux incidents critiques qui ont été définis. Nous élaborons aussi les transcriptions des entretiens relatifs à ces situations. Nous inférons les 
connaissances des enseignants en triangulant toutes les données issues des vidéos et des transcripts ${ }^{2}$.

\section{RÉSULTATS}

Nous présentons maintenant quelques résultats de notre recherche. Nous nous centrons sur un seul enseignant en choisissant des extraits qui nous paraissent représentatifs. Nous analysons un enseignement mis en œuvre par Henri pendant deux années consécutives. Les élèves doivent mesurer le poids d'un objet avec un dynamomètre puis apprendre son principe de fonctionnement. Nous en présentons une analyse du point de vue de la didactique des sciences puis de la didactique professionnelle avant de discuter nos résultats.

\section{Analyse d'un point de vue de la didactique des sciences}

\section{Première année d'observation}

Notre analyse s'appuie ici sur trois extraits du début de la première séance sur « poids et masse » mise en œuvre par Henri : la situation-problème, une activité de mesure du poids et une application.

\section{Mesure du poids d'un objet}

Henri débute la séquence par l'analyse d'une planche de bande dessinée des aventures de Tintin tirée de l'album « on a marché sur la Lune ${ }^{3} »$. Sur cette planche, nous voyons un des héros Dupont, sur la Lune, sauter une crevasse et retomber beaucoup plus loin que prévu. Dans les entretiens, l'enseignant justifie ce choix : son but est double. D'une part, il souhaite intéresser les élèves pour s'assurer de leur adhésion au cours, le plus longtemps possible. Par conséquent, cette planche de B.D. est un choix stratégique. D'autre part, cette analyse doit lui permettre de mettre en évidence le poids d'un corps. Henri en donne une définition, à l'oral, sans qu'elle soit l'objet d'une construction conjointe avec les élèves. Puis, ils doivent mesurer le poids d'objets présents dans leur trousse ou dans leur cartable, à l'aide d'un dynamomètre qu'ils utilisent pour la première fois.
Le problème de la mesure du poids est dévolu aux élèves à travers la découverte du dynamomètre que le professeur sait n'opposer aucunes difficultés dans ce cas. Il ne donne pas de consigne précise ni de protocole à suivre. L'enseignant n'intervient que « sur demande » ou s'il constate des difficultés. Les élèves observent, décrivent et manipulent à vide le dynamomètre. Ils testent les propriétés d'élasticité du ressort, la relation entre l'allongement, le repère et la graduation. Ils réinvestissent des savoirs sur la prise de mesure : ils placent le repère au niveau de l'œil pour éviter les erreurs de parallaxe dans la lecture de la valeur du poids de l'objet. Certains élèves testent les limites de l'appareil dans le cas d'une masse trop importante ou trop faible en changeant d'objet. Pour nous, ils font preuve d'une attitude scientifique dans le sens où ils évaluent le principe de fonctionnement du dynamomètre avant d'y suspendre un objet et d'effectuer une mesure. Toutes ces expériences, interprétées en termes d'interaction, constituent le référent empirique. Les élèves mobilisent des connaissances phénoménotechniques, c'est-à-dire des savoir-faire instrumentaux qui permettent d'effectuer correctement une mesure et des connaissances phénoménographiques c'est-à-dire des connaissances empiriques liées à l'utilisation pratique du dynamomètre.

Le principe de fonctionnement d'un dynamomètre

Henri s'appuie sur le référent empirique constitué précédemment pour mettre en évidence le principe de fonctionnement du dynamomètre. L'échange se fait en grand groupe.

Extrait $\mathrm{n}^{\circ} 1 \mathrm{du}$ transcript de l'activité de mesure ( $\mathrm{H}=$ Henri, $\mathrm{C}=$ Charlotte, $\mathrm{Es}=$ plusieurs élèves $)$

$\mathrm{H}$ : / Qu'est-ce qui fait ce ressort ? ((brouhaha)) Charlotte il monte il descend oui ? au moment où on suspend quelque chose

$\mathrm{C}:$ Il descend

$\mathrm{H}$ : / Et il est toujours étiré de la même manière ou pas?

Es : Non

$\mathrm{H}$ : Non ça dépend de quoi ça son étirement?

$\mathrm{C}:$ Du poids

$\mathrm{H}$ : Du poids de l'objet donc euh plus l'objet /

C : Est lourd

$\mathrm{H}$ : Plus il a un poids important plus il est étiré voilà vous avez le principe de cet appareil qu'on appelle un dynamomètre ((h écrit au tableau)) 
Cet échange porte sur des éléments qui appartiennent au registre du référent empirique à travers une connaissance phénoménographique de l'élève sur le dynamomètre (le ressort descend au moment où l'on suspend quelque chose, il n'est pas étiré toujours de la même manière). Henri s'appuie sur cette connaissance pour basculer dans le registre des modèles (l'étirement dépend du poids de l'objet). Cette mise en relation entre ces deux registres lui permet de conclure sur le principe de l'appareil de mesure et d'en justifier l'utilisation. Nous observons que l'enseignant justifie son étirement par l'action du poids, alors que l'élève interrogée fait référence à « lourd » c'est-à-dire à la masse. La tentative de passage dans le registre des modèles échoue.

\section{Application à un dynamomètre circulaire}

Henri propose de mesurer le poids d'un objet avec un autre type de dynamomètre. Il s'agit d'un dynamomètre à ressort circulaire dont on ne voit pas les éléments mécaniques qui le compose. Il est aimanté au tableau. Le professeur décrit son fonctionnement et y suspend une paire de ciseaux. Puis, il demande à un élève de venir lire le poids.

\section{Extrait $\mathrm{n}^{\circ} 2$ du transcript de l'activité de mesure}

$\mathrm{H}$ : Bon///le ciseau pèse

$\mathrm{E}:$ Zéro virgule quatre

$\mathrm{H}$ : Zéro virgule quatre

E : Newton

$\mathrm{H}$ : Newton / euh plutôt que pèse zéro virgule quatre newton qu'est-ce qu'on aurait pu dire d'autre

E : A une masse de

$\mathrm{H}$ : A une masse de vous êtes d'accord

Es : Oui

$\mathrm{H}$ : De quoi on parle là on parle de la masse

Es : Non du poids

$\mathrm{H}$ : Pour l'instant on ne mélange pas tout on parle du poids depuis le début du cours

Cette application n'était pas prévue par Henri. Il dit, dans les entretiens, la proposer pour, d'une part, répondre aux difficultés à différencier le poids et la masse et, d'autre part, vérifier qu'ils savent tous mesurer le poids d'un objet. La mesure ne pose pas de problème à l'élève. Par contre, l'amalgame entre le poids et la masse est toujours présent.

La masse et le poids sont confondus

À travers ces deux extraits, nous remarquons que les élèves ne savent pas s'ils mesurent le poids ou la masse d'un corps avec un dynamomètre. Au-delà de la mesure, nous pensons qu'ils ne distinguent pas ce qui est une propriété d'un morceau de matière, la masse $^{4}$, de son interaction avec la Terre, le poids. Il nous semble que cette difficulté provient du fait qu'il n'y a pas de mise en relation entre les événements et les propriétés des interactions dans leurs réponses aux questions du professeur. Lemeignan et WeilBarais (1993) soulignent que les concepts « agir » et « objet» doivent être admis pour permettre cette mise en relation. Ils font partie des descripteurs qui, selon Genzling et Pierrard (1996), sont nécessaires dans les activités de modélisation. Cela signifie que la Terre, la main, le ressort et le corps suspendu doivent être définis comme des objets et décrits selon les interactions que chacun entretient avec les autres. Lemeignan et Weil-Barais suggèrent de distinguer cette démarche qui vise à définir le concept d'interaction de la modélisation connexe qui est déterminée par les caractéristiques de l'interaction en termes de direction, sens, importance, réciprocité. Dans la séance observée, Henri n'a pas mis les élèves dans la situation de construire le modèle du poids comme un modèle interaction à partir des deux démarches conjointes suggérées par Lemeignan et Weil-Barais. Cela expliquerait les difficultés des élèves à différencier le poids de la masse d'un objet et à montrer le principe de fonctionnement du dynamomètre.

\section{Deuxième année d'observation}

L'introduction de l'activité expérimentale est modifiée entre les deux années. Par contre, le début de la séance ne change pas : l'enseignant s'appuie sur l'étude de la même bande dessinée pour définir le poids. Mais, contrairement à la première année, cette définition est construite conjointement avec les élèves et constitue la première trace écrite de la leçon.

\section{Définition du poids}

Henri a identifié deux dysfonctionnements à l'issue de la première année : d'une part, il dit avoir construit le cours au tableau trop tard (au bout de trente-cinq minutes, dans la première séance) et, d'autre part, il a constaté que le concept de gravitation (vue au chapitre précédent) n'était pas acquis pour beaucoup d'élèves. Or, il s'appuie sur ce concept pour définir le poids et introduire sa mesure. Par conséquent, la deuxième année, il a insisté un 
peu plus sur le concept de gravitation et il met en place la trace écrite au bout de deux minutes de la première séance. Les élèves ont noté la définition suivante : « on appelle poids d'un corps l'attraction d'une planète sur le corps ${ }^{5} »$. Pour construire cette définition, ils ont fait « fonctionner » le modèle de l'interaction gravitationnelle pour expliquer pourquoi Dupont est surpris par la longueur de son saut sur la Lune.

Extrait $n^{\circ} 3$ du transcript de la séance 1/année 2 concernant l'étude de la B.D (E1 = élève 1)

E1 : Il est allé aussi loin/c'est à cause de la gravitation de la Lune

$\mathrm{H}$ : Et alors qu'est-ce que tu veux dire par là c'est à cause de la gravitation de la Lune?

E1: La Lune euh elle attire moins les objets que la Terre

Dans cet extrait, l'élève El précise deux choses : d'une part, que chacune des planètes attire un objet et, d'autre part, que l'attraction de la Lune sur l'objet serait moindre que celle de la Terre. On remarque que sa réponse est formulée dans un cas général grâce à la relance de l'enseignant. Puis, Henri présente le dynamomètre et sa fonction en relation avec la définition du poids donnée précédemment alors que, la première année, il avait demandé aux élèves si le poids se mesurait.

\section{Conséquences sur la tâche des élèves}

L'évolution de l'introduction de l'activité expérimentale n'a pas eu d'influence sur l'activité de mesure suivante. Henri n'a rien changé, ni dans son organisation ni dans sa conduite ni dans le matériel utilisé, au cours des deux années. Cependant, même si l'énoncé de la tâche des élèves est inchangé, elle n'est plus tout à fait la même. En effet, ils savent, avant de faire des expériences, que le dynamomètre mesure l'attraction de la Terre sur l'objet. Voici deux extraits qui nous paraissent représentatifs :

Extrait $\mathbf{n}^{\circ} 4$ de l'activité de mesure (E2 = élève 2)

$\mathrm{H}$ : Est-ce que le ressort s'étire toujours de la même manière Manu ?

E2 : Bin non ça dépend du poids de l'objet
Dans ce tour de parole, l'enseignant se place au niveau du registre empirique. C'est sous son influence que l'élève E2 explique le phénomène

\section{Extrait $n^{\circ} 5$ de l'activité de mesure}

H : (..) C'est très bien donc plus le poids de l'objet sera important /

E2 : Plus le euh le ressort

$\mathrm{H}:$ Le ressort

E2 : Va s'étirer

empirique en faisant référence au modèle du poids et à sa définition construite précédemment.

Henri se situe au niveau du registre du modèle. Sous son influence, l'élève opère une projection du modèle sur le référent empirique. Il décrit ce qui se passerait si on augmentait le poids. Par conséquent, il développe une connaissance phénoménologique qui élargit le référent empirique au dynamomètre.

Les réponses des élèves satisfont Henri. Pour lui, ils ont bien compris le principe de fonctionnement du dynamomètre. Donc, il ne juge pas utile de proposer une application au dynamomètre à ressort circulaire, contrairement à la première année. En effet, nous observons les élèves mis en situation, par les questions du professeur, de définir les objets en interaction ainsi que les caractéristiques de cette interaction. Ils précisent l'interaction entre Dupont et la Lune; ils donnent des caractéristiques de cette interaction en termes de sens, de réciprocité et d'importance par comparaison avec la Terre. Dans l'extrait $n^{\circ} 3$, nous observons une partie de ce travail mené en grand groupe par l'enseignant. De fait, Henri a pu associer les élèves à la construction de la définition du poids qui leur permet d'accéder au modèle de type interaction (Lemeignan \& Weil-Barais, 1993). En conséquence, ils n'ont pas eu de difficultés à expliquer le principe de fonctionnement du dynamomètre.

Nous allons maintenant examiner ce début de séquence sous l'angle de la didactique professionnelle. Nous analysons une organisation de l'activité de l'enseignant à travers l'identification des schèmes en jeu. Nous en déterminons les éléments constitutifs en identifiant les connaissances professionnelles dans les invariants opératoires. Puis, nous discutons de l'articulation des schèmes entre eux dans l'organisation de l'activité des enseignants. 


\section{Analyse d'un point de vue de la didactique professionnelle}

Notre méthodologie basée sur une confrontation des professeurs à leur action et des entretiens de type autoconfrontation produit des résultats qui questionnent la représentation du schème. Il nous semble nécessaire de le décrire suivant six éléments au lieu de quatre dans la définition originale. Pour cet aspect de notre travail, nous faisons référence à la Grounded theory (Glaser \& Strauss, 1967). Selon ce point de vue, l'analyse des données fait ressortir des catégories qui nous amènent à des propositions d'évolution de la théorie, que nous présentons ci-dessous.

\section{Une nouvelle description du schème}

Les résultats de notre travail nous permettent d'étendre les quatre composantes du schème à six éléments articulés différemment. Nos analyses portent sur des vidéos de classe, dans lesquelles nous voyons les enseignants en action, et sur des vidéos des entretiens en auto-analyse simple et croisée. Or, si dans le cadre théorique de référence, les indices sont subordonnés aux règles de prise d'informations, lors des entretiens, les enseignants présentent ces indices comme des éléments majeurs : les professeurs commentent la relation qui existe entre les indices qu'ils prennent dans la classe et la conduite de leur action. De fait, dans notre description de l'activité des professeurs, nous explicitons les indices prélevés dans l'action. En conséquence, nous ajoutons les indices comme élément constitutif du schème. Par contre, les règles de prise d'informations ne sont pas explicitées dans les entretiens. Cet aspect peut être une conséquence de notre méthodologie puisque nous nous centrions sur des incidents critiques fortement connectés au savoir en jeu. De fait, nous avons décidé de ne pas les énoncer.

L'analyse de nos données fait apparaître la nécessité de rendre visibles les anticipations, en les dissociant du but et des sous-buts. Il s'agit ici des anticipations que l'enseignant a du but de son activité, c'est-à-dire du résultat de cette activité sur les élèves de manière individuelle ou collective. Elles nous permettent d'observer ses actions, face à des réponses ou des attitudes d'élèves. La description de ce qui se passe dans la classe est affinée par comparaison avec ces anticipations qui nous aident aussi, lors des entretiens, à analyser l'activité de l'enseignant, notamment du point de vue des régulations qu'il met en place. Par conséquent, nous décrivons ici le schème suivant six éléments : un but et des sous-buts, des anticipations, des inférences, des indices, des règles d'actions, des invariants opératoires (concept-en-acte et théorème-en-acte).

Prenons un exemple pour caractériser un schème en renseignant ses six éléments. Henri introduit l'activité expérimentale dans laquelle les élèves doivent mesurer le poids d'un objet, par la question : «Est-ce que l'on peut mesurer le poids ? ». Nous présentons les éléments du schème modélisant l'organisation de son activité dans un tableau (tableau 1, ci-dessous). Nous le notons schème B.1. pour indiquer qu'il s'agit du schème B la première année. La colonne de gauche indique les éléments constitutifs du schème, la colonne de droite propose ce que l'enseignant fait ou pense en situation.

Tableau 1. Année 1. Schème B.1 «Introduction de l'activité expérimentale »

\begin{tabular}{|l|l|}
\hline \multicolumn{2}{|l|}{ Schème B.1 : introduction de l'activité expérimentale } \\
\hline But & Mettre en évidence que le poids se mesure \\
\hline Anticipation & J'attends que les élèves répondent par l'affirmative en donnant son unité \\
\hline Inférences & $\begin{array}{l}\text { Si les élèves ne répondent pas alors je pose la question en nommant un élève. } \\
\text { Si je sens des doutes alors je demande si quelqu'un pense que non. } \\
\text { Si un élève répond par l'affirmative je lui demande pourquoi. } \\
\text { Si les élèves font référence au document « Newton et la gravitation » alors je poursuis le dérou- } \\
\text { lement du cours. }\end{array}$ \\
\hline Indice & Les élèves répondent à la question. \\
\hline Règle d'action & Demander aux élèves si le poids se mesure \\
\hline Invariants opératoires & $\begin{array}{l}\text { Je sais que le poids se mesure avec un dynamomètre. } \\
\text { Lunité de mesure du poids est le newton de symbole N. } \\
\text { La réponse à la question est présente dans le document « Newton et la gravitation » déjà étudié. } \\
\text { Poser la question de la mesure du poids permet d'introduire le dynamomètre. }\end{array}$ \\
\hline
\end{tabular}


Le contenu concernant le but, les anticipations, l'indice, la règle d'action sont énoncés dans les entretiens par Henri. Par contre, nous déduisons les inférences de la vidéo de classe car elles ne sont pas explicitées par le professeur. Les invariants opératoires sont déduits à la fois des entretiens et des vidéos de classe. Pour plus de précision, nous reformulons tous ces propos lors de la mise en tableau.

Nous relevons les connaissances de l'enseignant dans l'invariant opératoire du schème B.1, à partir de l'étude des théorèmes-en-acte et des concepts-enacte. Henri tient pour vrai que le poids se mesure avec un dynamomètre, son unité est le newton. Il fait la proposition de débuter la séance par une question sur la mesure pour introduire le dynamomètre car il sait que cette question a été vue à la séance précédente dans l'étude du texte « Newton et la gravitation ». Les concepts-en-acte n'apparaissent pas clairement dans notre description du schème B.1. Ils ont une fonction de prélèvement de l'information dans la situation afin d'orienter l'action. Il nous est difficile de les identifier à partir de l'action des enseignants ou des entretiens.

Quels sont les types de connaissances identifiées dans cette organisation ? Dans cet exemple, nous identifions des connaissances de type SMK (le poids se mesure avec un dynamomètre, l'unité de mesure du poids est le newton de symbole $N$ ). Toutes les autres connaissances sont spécifiques à l'enseignement sur « poids et masse ». Pour nous, ce sont des PCK sur le matériel pédagogique (le dynamomètre) et sur les stratégies (la question de la mesure permet d'introduire le dynamomètre). Mais, il y a aussi une connaissance sur le savoir construit par les élèves dans une tache passée (la réponse à la question est présente dans le document "Newton et la gravitation " déjà étudiée). C'est une PCK sur les élèves dans la sous-catégorie « les prérequis nécessaires à l'apprentissage d'une notion ».

Regardons comment s'organise l'activité d'Henri en ce début de séance. Il s'agit d'identifier les schèmes du professeur et de discuter leur articulation.

\section{Organisation de l'activité d'Henri : cas de la première année}

Nous distinguons deux parties dans l'organisation de l'activité mise en œuvre par Henri. La première concerne l'activité expérimentale et la seconde un travail sur le principe de fonctionnement du dynamomètre avec une application. Dans la première partie les élèves sont en groupes; dans la seconde partie l'enseignant interagit avec la classe entière. Nous décrivons l'organisation de l'activité par quatre schèmes notés A, B.1, C et D (figure 3).

Figure 3. Organisation de l'activité sur la mesure du poids d'un objet

Schème $A$ : Mesure du poids d'un objet

Schème B.1 : Introduction de l'activité expérimentale

Incident critique : Le poids est une grandeur mesurable

Sous-schème 1 : Suspension de la tâche

Sous-schème 2 : Lecture du document Newton et la gravitation

Sous-schème 3 : Retour au schème B.1

Schème $\mathrm{C}$ : Principe de fonctionnement du dynamomètre

Schème $D$ : Application à autre type de dynamomètre 
Les schèmes A et B.1 concernent l'activité expérimentale; les schèmes $C$ et $D$ rendent compte de l'organisation de l'activité sur le principe de fonctionnement du dynamomètre et une application. Selon la théorie de Vergnaud, un schème peut comporter un but et des sous-buts ou bien il peut piloter l'utilisation de plusieurs autres schèmes. Autrement dit, les schèmes se composent et se décomposent hiérarchiquement. Mais à partir de quel critère peut-on modéliser l'organisation de l'activité des enseignants en schème et sous-schèmes ou un but en sous-but ?

Nous faisons le choix de prendre comme critère les anticipations du but du professeur dans l'activité. Lorsque nous identifions un changement de but, nous comparons les anticipations de chacun des buts. Si nous constatons que les anticipations restent les mêmes, alors il n'y a pas de nouveau schème : le schème « initial » s'écrira avec un but et des sousbuts correspondants à chaque nouvelle règle d'action. Par contre, si les anticipations ne sont pas les mêmes alors il y a un nouveau schème. Nous considérons, alors, que ce nouveau schème est un sous-schème du schème « initial ». Dans notre exemple (figure 3), les anticipations du schème $\mathrm{A}$ " mesure du poids d'un objet » ne sont pas identiques à celles du schème B.1 « introduction d'une activité expérimentale ». En effet, d'un côté, Henri attend que les élèves s'approprient le dynamomètre pour effectuer une mesure et, de l'autre, il attend que les élèves répondent par l'affirmative à la question posée. Donc, le schème B.1 est un sous-schème du schème $A$. De la même manière, nous disons que le schème $\mathrm{D}$ « application à autre type de dynamomètre » est un sous-schème du schème $C$ « principe de fonctionnement du dynamomètre ». De fait, le schème A pilote le schème B.1 et le schème $C$ pilote le schème $\mathrm{D}$.

Nous allons maintenant caractériser les régulations opérées par le professeur suite à des incidents critiques identifiés au cours des entretiens.

\section{Relation entre des connaissances professionnelles et la régulation rétroactive de l'activité en classe}

Nous présentons ici trois exemples d'incidents critiques à l'origine de trois boucles de régulations rétroactives de l'activité : une boucle courte, une boucle longue et une boucle « changement de schème ». Nous décrivons chacune de ces boucles en faisant la relation avec les connaissances professionnelles mobilisées par Henri. Pour cela, nous étudions les invariants opératoires et les inférences du schème.

\section{Une boucle courte de régulation}

La première année, Henri introduit l'activité expérimentale dans laquelle les élèves doivent mesurer le poids d'un objet par la question : "Est-ce que l'on peut mesurer le poids ? » (§ 3.2.1). Henri dit, dans l'entretien, son étonnement devant la difficulté pour les élèves à répondre. En effet, il dit avoir déjà travaillé sur cette question, au cours précédent, en étudiant le texte Newton et la gravitation qui présente les travaux de Newton sur ce sujet. L'enseignant reprend alors l'étude de ce texte. Elle n'était pas prévue. Il s'agit d'un incident critique que nous nommons « le poids est une grandeur mesurable». Le professeur acquiert alors une nouvelle PCK sur les élèves «les élèves ne savent pas que le poids est une grandeur mesurable».

Henri a opéré une régulation que nous représentons par une boucle courte. Nous la décrivons suivant trois sous-schèmes du schème B.1 (figure 3) car les anticipations de chacun des buts changent pendant l'adaptation de l'enseignant aux difficultés des élèves à répondre à la question. En effet, le premier but est de mobiliser la classe sur la question posée. Le second est de trouver la réponse à la question en reprenant la lecture du texte Newton et la gravitation. Le troisième but du professeur est de revenir au but initial qui est d'introduire l'activité expérimentale de mesure du poids d'un objet. Au cours de cette régulation, Henri mobilise des connaissances professionnelles. Nous identifions une PK sur la classe lorsqu'Henri mobilise tous les élèves sur la question et dans sa gestion de la lecture du document. Nous déterminons aussi une SMK sur le concept de poids et sa mesure et une PCK sur les élèves «les élèves ont déjà étudié le document Newton et la gravitation au cours précédent». Elle appartient à la sous-catégorie « Les prérequis nécessaires pour l'apprentissage d'une notion » dans notre modèle de référence. Cette régulation permet à Henri d'atteindre son but en opérant de proche en proche (Pastré, 1997). Elle est le résultat d'un écart entre l'anticipation du schème et les indices pris dans l'action.

Cet incident critique est une des causes de la modification du début du cours d'Henri l'année 
suivante. En effet, à la fin du chapitre sur «poids et masse ", la première année, il a identifié deux dysfonctionnements dans la première séance : d'une part, il dit avoir construit le cours au tableau trop tard (au bout de trente-cinq minutes) et, d'autre part, il a constaté que le concept de gravitation n'était pas acquis pour beaucoup d'élèves. Or, il s'appuie sur ce concept pour définir le poids et introduire sa mesure. À l'issue de cette analyse, le professeur a construit trois nouvelles connaissances de type PCK sur les élèves : le concept de gravitation n'est pas acquis pour beaucoup d'élèves; la définition du poids donnée à l'oral n'est pas comprise; les élèves ne savent pas que le poids est une grandeur mesurable. Du point de vue du modèle de Magnusson et al., les deux premières appartiennent à la sous-catégorie «La connaissance des domaines pour lesquels les élèves ont des difficultés » et la troisième « Les pré-requis nécessaires pour l'apprentissage d'une notion $»$.

Ces trois nouvelles connaissances sont à l'origine de changements assez profonds dans la séquence. D'abord, Henri souligne avoir insisté beaucoup plus sur le concept de gravitation au chapitre précédent : « Il me paraît nécessaire de leur faire comprendre [...] d'insister sur le fait que quelque chose a une masse il est attiré par la terre c'est la gravitation j'avais beaucoup insisté là-dessus ». Nous avons montré, au paragraphe 3.1.2, comment le professeur a construit la définition du poids conjointement avec les élèves et comment il a modifié l'introduction de l'activité expérimentale en présentant le dynamomètre et sa fonction en relation avec la définition du poids.

\section{Une boucle de régulation "changement de schème »}

Nous représentons la réorganisation de l'activité d'Henri par la modification du schème $B$ « Introduction de l'activité expérimentale », contrairement au schème A " Mesure du poids d'un objet » (figure 4). Identifions précisément les éléments du schème $\mathrm{B}$, noté schème B.2. (pour la deuxième année), qui ont évolué (tableau 2).

Le but, la règle d'action et les invariants opératoires ont changé au cours des deux années. Pour nous, ceci est caractéristique d'une boucle de régulation « changement de schème ». L'activité d'Henri est modifiée car il présente le dynamomètre comme « l'appareil qui permet de mesurer l'attraction exercée par une planète sur un corps ». De fait, il s'agit d'un changement de théorème-en-acte. Le but et la règle d'action sont corrélés avec le nouveau théorème-enacte. Du point de vue des connaissances, c'est une PCK sur les stratégies qui correspond à ce changement de théorème-en-acte. Elle indique le choix que fait l'enseignant pour articuler la définition du poids et sa mesure.

Nous n'avons pas relevé d'incident critique au cours de l'introduction à l'activité expérimentale la deuxième année. Nous en déduisons que le déroulement prévu par Henri est bien celui qui a été réalisé. C'est pour cette raison qu'il n'a pas jugé utile de proposer une application à un autre type de dynamomètre. Nous notons (figure 4) que l'incident critique de la première année s'est transformé en un incident prévu la deuxième année. Henri dit avoir prévu une relecture du document la deuxième année. Le professeur a opéré une boucle longue de régulation.

Tableau 2. Année 2. Changement de schème B.2 «Introduction de l'activité expérimentale »

\begin{tabular}{|l|l|}
\hline Schème B.2 : Introduction de l'activité expérimentale \\
\hline But & Introduire un nouvel appareil de mesure : le dynamomètre. \\
\hline Anticipation & J'attends que les élèves écoutent. \\
\hline Inférence & Si aucun élève ne pose de question alors je poursuis. \\
\hline Indice & Les élèves sont attentifs. \\
\hline Règle d'action & Présenter le dynamomètre et sa fonction. \\
\hline $\begin{array}{l}\text { Invariants } \\
\text { opératoires }\end{array}$ & $\begin{array}{l}\text { Je sais que le poids se mesure avec un dynamomètre } \\
\text { L'unité de mesure du poids est le newton de symbole N. } \\
\text { Je donne le nom de l'appareil de mesure et sa fonction en relation avec la définition } \\
\text { construite précédemment }\end{array}$ \\
\hline
\end{tabular}


Figure 4 : Boucle à changement de schème

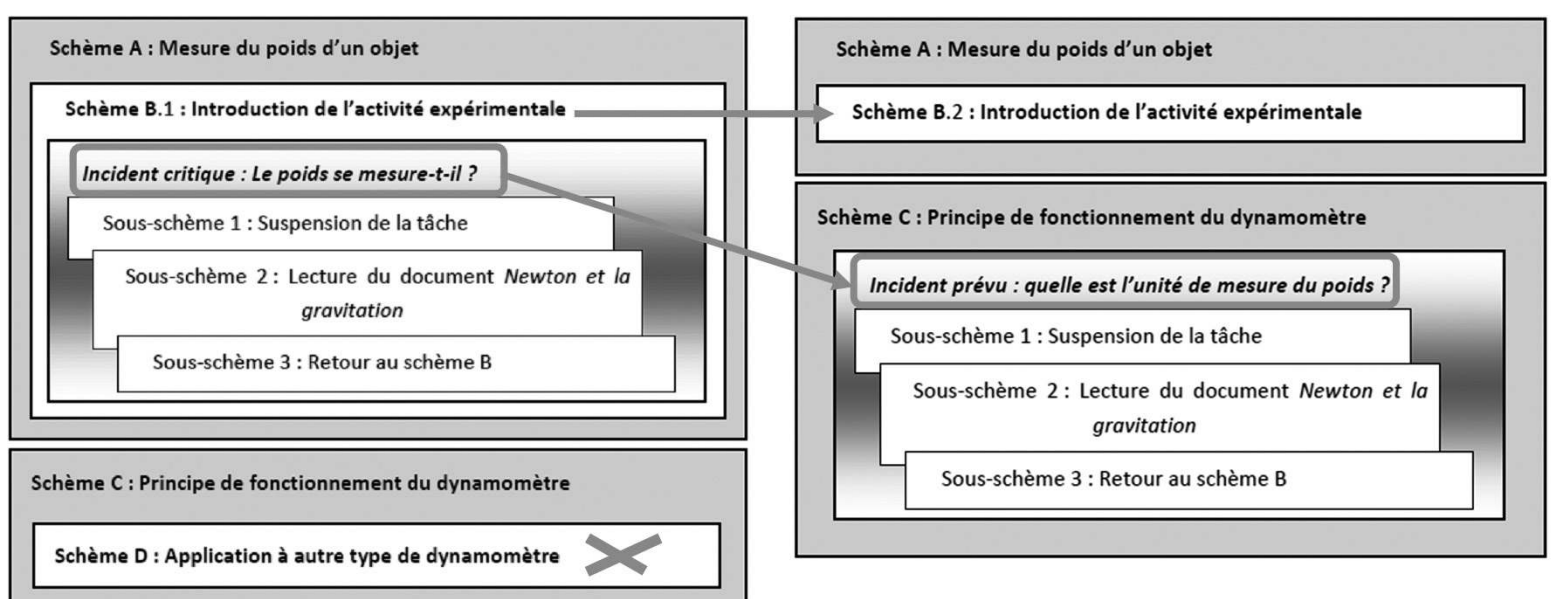

Année 2

\section{Année 1}

\section{Une boucle longue de régulation}

Suite à l'incident critique « le poids est une grandeur mesurable », Henri a acquis une nouvelle PCK que nous avons notée "les élèves ne savent pas que le poids est une grandeur mesurable $»(\& 3.3 .1)$. Cette nouvelle connaissance conduit le professeur, la deuxième année, à demander aux élèves de lire le dernier paragraphe du texte Newton et la gravitation pour trouver l'unité du poids. Cette étude du texte est prévue par Henri. Elle est faite après une première série de mesures avec le dynamomètre (figure 4). Par conséquent, elle ne se trouve pas au même endroit du cours entre les deux années. C'est une conséquence de la réorganisation de son activité que nous avons décrite au paragraphe précédent.

De fait, l'incident critique de la première année s'est transformé en un incident prévu la deuxième année. Ce dernier garde la même organisation que l'incident critique. Nous identifions une nouvelle inférence "si les élèves ne connaissent pas l'unité de mesure du poids je leur demande de lire le document Newton et la gravitation » qui s'appuie sur un nouvel invariant opératoire "je sais que les élèves ne savent pas que le poids est une grandeur mesurable » dans le schème $\mathrm{C}$. La capitalisation de ces deux éléments du schème est, selon nous, caractéristique d'une boucle longue de régulation.

Nous observons que l'enseignant a appris sur le seul fait d'agir en situation. À partir d'un incident critique, il capitalise des inférences et des invariants opératoires : l'activité du professeur possède un versant constructif et un versant productif (Samurçay $\&$ Rabardel, 2004). Nous remarquons que cette construction est très locale puisqu'elle est en relation avec une notion précise à enseigner.

\section{DISCUSSION}

Nous allons, maintenant, discuter les éléments d'analyse présentés dans cette étude. Nous enrichissons nos propos en nous appuyant sur le cas de Florence. Nous débutons par les connaissances professionnelles mobilisées par les enseignants. Nous comparons nos résultats à ceux de notre modèle de référence (Magnusson et al., 1999) et nous évoquons certaines limites. Puis, nous présentons une évolution des connaissances professionnelles à travers des mécanismes d'acquisition de nouvelles connaissances. Nous analysons des conséquences sur les réorganisations de l'activité des professeurs. Enfin, nous discutons des intérêts à croiser les deux didactiques pour cette étude.

\section{Les connaissances professionnelles des enseignants}

Le modèle du schème que nous avons étendu à six composantes nous permet de comprendre comment 
l'activité d'Henri est organisée. Nous apercevons les étapes que doit franchir l'enseignant pour atteindre son but. Le lien entre les connaissances professionnelles et l'organisation de l'activité se fait par les invariants opératoires du schème et, plus précisément, à partir des théorèmes-en-acte. Lors de l'introduction à l'activité expérimentale, nous relevons des connaissances disciplinaires, des SMK, et des connaissances spécifiques à l'enseignement du poids, des PCK. Nous identifions des PCK dans trois composantes: une connaissance des stratégies d'enseignement, des connaissances sur les élèves et sur les programmes (\$3.2.1). Nos observations nous amènent à décrire l'organisation de l'activité de Florence comme celle d'Henri (figure 3). Lors de l'introduction à l'activité expérimentale, nous notons des SMK sur le poids et sa mesure et des PCK sur les stratégies (je donne le nom de l'appareil de mesure et sa fonction en relation avec la définition construite précédemment), sur les programmes (l'utilisation du matériel pédagogique : les dynamomètres opaques à ressort circulaires et transparents à ressort longitudinal) dans l'invariant opératoire du schème de l'enseignante.

Nous avons observé Florence mobiliser un autre type de connaissance présent dans notre modèle de référence : les connaissances sur le contexte d'enseignement, notées KofC. Elles sont mobilisées dès qu'il s'agit d'adapter l'enseignement aux spécificités (contexte socio-culturel, hétérogénéité du groupe, etc.) d'une classe ou d'un établissement. Florence a modifié sa préparation initiale entre les deux collèges, que nous notons $\mathrm{SM}$ et $\mathrm{P}$, dans lesquels elle enseigne. Notre exemple est extrait de l'activité qui précède la mesure du poids d'un objet. Dans le collège SM, elle note les "caractéristiques » (définition, unité et appareil de mesure) du poids et de la masse à partir d'un texte extrait du mensuel Science EVie Junior ${ }^{6}$. C'est-à-dire qu'elles se succèdent dans des paragraphes distincts et numérotés. Son but est que les élèves perçoivent ce qui distingue ces deux grandeurs. Au fil de son cours, elle se rend compte du manque de clarté de sa présentation : «j'ai trouvé que en le faisant même en regardant le tableau ((le support au mur)) même de loin que ça faisait moins heu / ben déjà la distinction poids masse n'est pas spécialement claire». Elle décide, pour le même cours dans le collège $\mathrm{P}$, de renseigner les « caractéristiques» du poids et de la masse dans un tableau. Elle évoque des causes qui expliqueraient les difficultés que ces élèves rencontrent régulièrement. L'enseignante dit qu'ils sont d'origines modestes, qu'ils ont moins de facilités pour faire leurs devoirs en rentrant le soir. La plupart habitent dans des fermes et « ils doivent aider le soir en rentrant à la maison». Elle ajoute que les cas difficiles sont plus nombreux dans ce collège. De fait, elle change régulièrement sa préparation en fonction des difficultés qu'elle perçoit lors de son enseignement dans le collège SM. À propos de l'enseignement de la mécanique, elle sait que les élèves du collège $\mathrm{P}$ ont rencontré des difficultés dans le chapitre précédent sur la gravitation.

Au cours de cette analyse, Florence mobilise des connaissances de types PCK sur les élèves et des KofC. Elle ajoute que les élèves au collège $P$ ont besoin d'un cours synthétique, plus facile à la relecture. Cette connaissance, que nous qualifions de pédagogique, notée PK dans le modèle de Grossman (1990), influence l'enseignement de Florence concernant la notion du poids et de la masse. La mise en tableau est un produit de cette influence.

Notre cadre d'analyse nous permet d'identifier les quatre types de connaissances présents dans le modèle de Magnusson et al., (1999). Nous montrons comment les SMK et les KofC se retrouvent mêlées aux PCK dans l'organisation de l'activité des enseignants. Cependant, il nous paraît difficile de distinguer les deux sous-catégories des PCK sur les stratégies de notre modèle de référence : les «Connaissances des stratégies dans l'enseignement des sciences » et "Connaissances sur des activités spécifiques à un sujet de science ». La frontière est difficile à placer car c'est la définition d'une stratégie qui est posée. Qu'est-ce qui est stratégique pour un enseignant? Est-ce la mise en place d'un dispositif particulier comme une expérience dans un cours ou de débuter une séquence en posant une question ou le choix d'un type de support comme une planche de BD ou est-ce le dispositif et la démarche qui y sont associés ? Si nous considérons que c'est le dispositif ou le type de support qui est stratégique, pour nous, le professeur mobilise une PK car un dispositif ou un support n'est pas spécifique à un sujet d'étude. Par contre, lorsqu'un enseignant utilise une analogie, il mobilise une PCK sur les stratégies. Il s'agit bien d'une démarche avec des connaissances en jeu qui vise à aider les élèves à comprendre un ou des phénomènes ou à conceptualiser des notions. De même, la frontière avec une PCK sur les élèves peut être mince. Prenons un exemple. Lorsque Florence reformule la tâche que doivent faire les élèves pour trouver la loi $P=m g$, elle emploie les 
termes «trouver une relation mathématique » au lieu de « montrer qu'il y a un lien ou qu'il n'y a pas de lien». Lors de cette régulation, le professeur mobilise-t-il une PCK sur les stratégies ou une PCK sur les élèves?

Une autre question se pose au vu de notre étude. Quel est l'impact des SMK dans la préparation de la classe et la mise en œuvre ? Cette question se pose car les connaissances disciplinaires sont difficiles à identifier. En effet, pendant les entretiens, les professeurs sont centrés sur leur action ou l'action des élèves dans la classe. De plus, dans le second degré, les enseignants sont disciplinaires. Ils ne cherchent pas à justifier " théoriquement » leurs choix lors de l'entretien en auto-analyse croisée : implicitement, ils considèrent que le collègue sait. Mais, dès qu'une discussion entre les deux enseignants s'intensifie nous voyons apparaître des SMK dans leurs justifications. Ils ne sont plus centrés sur la vidéo. Nous soulignons cet aspect méthodologique car il nous permet d'identifier les SMK des enseignants et de voir comment, à partir des différents types de connaissances, les professeurs opèrent leurs choix lors de la préparation ou dans l'action. Par exemple, Florence propose à Henri d'utiliser un dessin animé comme situation-problème dans lequel Tintin, Milou et le capitaine Haddock sont en état d'impesanteur à l'intérieur d'une fusée en orbite autour de la Terre. Elle dit vouloir définir le poids en présentant un cas d'absence de pesanteur. Henri lui démontre qu'il ne s'agit pas d'une absence de pesanteur ${ }^{7}$ : «attention y'a la fameuse vitesse qui compense // le poids donc euh...le danger c'est de dire qu'il n'y a pas de poids». Cet exemple nous montre la présence implicite de connaissances de type SMK dans les choix que font les enseignants pour la classe.

Comme nous l'avons montré précédemment, notre description de l'organisation de l'activité nous permet d'identifier les connaissances professionnelles des enseignants dans les invariants opératoires. Mais, il arrive parfois qu'elles se retrouvent aussi dans les inférences du schème de l'enseignant. Par exemple, dans le cas du schème $C$ (figure 4 ) nous identifions des SMK sur l'unité de mesure de l'intensité du poids et sur le rôle du ressort dans la mesure. Nous les notons : "Si un élève parle de graduation je lui demande en quelle unité elle est exprimée et comment on le sait »; «Si un élève ne parle pas d'étirement du ressort alors je pose la question à la classe ». Donc, la régulation de l'activité en situation peut nécessiter, pour l'enseignant, de s'appuyer sur des connaissances scientifiques. De fait, nous pouvons distinguer les connaissances nécessaires pour réguler l'activité, des connaissances utiles pour prélever et sélectionner l'information pertinente. Nous observons que les schèmes gèrent à la fois la succession des actions, des prises d'informations pour poursuivre, et des contrôles. De fait, pour étudier le lien entre les connaissances professionnelles et l'organisation de l'activité, il nous paraît nécessaire d'analyser les deux composantes du schème convoqué dans l'action : les invariants opératoires et les inférences.

\section{Évolution des connaissances professionnelles des enseignants : conséquences sur les réorganisations de l'activité}

Nous identifions l'acquisition de nouvelles connaissances à partir d'incidents critiques relevés par les professeurs. Elles sont de type PCK sur les élèves dans les sous-catégories «Connaissance des domaines pour lesquels les élèves ont des difficultés » et «Les prérequis nécessaires pour l'apprentissage d'une notion $»$. Ces nouvelles connaissances sont très locales (Morge, 2003). Elles sont à l'origine des régulations opérées par les enseignants suite aux incidents critiques.

Notre analyse montre qu'une boucle courte de régulation n'apparait pas à chaque incident critique. Par exemple, dans le cas de Florence, elle ne juge " pas claire » la distinction du poids et de la masse (\& 4.1). Pour autant, nous ne relevons pas de régulation visant à modifier sa présentation en paragraphes dans le collège SM. C'est après son cours que le professeur trouve la solution qu'elle mettra en œuvre dans le collège $P$. Il en est de même à propos des trois PCK sur les élèves acquises par Henri et qui sont à l'origine de la boucle de régulation « changement de schème » (décrite en 3.3.2). En effet, seul l'incident critique « le poids est une grandeur mesurable » est identifié par l'enseignant. Pourtant les deux autres PCK sur les élèves « le concept de gravitation n'est pas acquis pour beaucoup d'élèves » et "la définition du poids donnée à l'oral n'est pas comprise » sont acquises sans que nous ne relevions une régulation de type boucle courte. Par conséquent, même si nous ne relevons pas de régulation opérée par un enseignant, une nouvelle connaissance peut être tout de même acquise. Celle-ci peut faire système avec d'autres nouvelles connaissances construites à différents moments de la séance ou de la séquence d'ensei- 
gnement pour participer à une régulation que nous décrivons par une boucle " changement de schème ».

En effet, c'est bien parce que ces trois PCK sur les élèves sont en relation les unes avec les autres qu'Henri peut changer sa situation d'entrée dans la séquence la deuxième année. Elles font système pour engendrer une boucle de régulation rétroactive de type « changement de schème ». Ces connaissances sont acquises dans la première partie de la première séance et permettent au professeur d'expliquer la confusion des élèves entre le poids et la masse qui persiste tout au long de la séquence. Ce rapport de « cause à effet " produit par le professeur nécessite sur les élèves tout au long de la séquence. Pour autant, des questions se posent : la sauvegarde de chaque nouvelle connaissance peut-elle se faire à une échelle de temps dépassant la séquence ? Est-ce seulement les nouvelles connaissances énoncées lors des entretiens par les enseignants qui font système?

Nous avons quelques éléments de réponse à ces questions. Par exemple, nous avons observé Florence dans la situation suivante durant les deux années de notre étude : un élève confond la masse et le volume. L'enseignante fait le choix de " corriger l'erreur» à chaque fois, mais elle ne donne pas la même réponse. La première année, elle parle de contenance pour montrer la différence entre le volume et la masse ; la deuxième année, elle fait référence au programme de la classe de cinquième où le volume et la masse sont définis. Elle dit dans l'entretien avoir construit cette réponse deux mois plus tard après l'incident, lors d'un cours en classe de cinquième. Nous voyons, dans ce cas, que la PCK sur les élèves "les élèves confondent la masse et le volume » est d'une durée qui dépasse la séquence. Elle permet au professeur de co-construire, avec les élèves, une réponse plus précise l'année scolaire suivante. Pareillement, nous pensons que des nouvelles connaissances construites tout au long de la séquence, comme par exemple lors des résultats d'évaluation, peuvent faire système avec celles construites auparavant pour engendrer une réorganisation de l'activité du professeur.

\section{Intérêts du croisement des deux didactiques pour décrire l'activité des enseignants dans une interaction professeur-élèves}

Pour nous le croisement des deux didactiques pour analyser l'interaction professeur-élèves présentent plusieurs intérêts. La didactique professionnelle nous permet de rendre compte de l'organisation et des changements d'organisation de l'activité, pour les notions effectivement enseignées. Nous y plaçons les connaissances professionnelles des professeurs et nous montrons leur impact au niveau de l'activité, de son organisation et de sa conduite. La didactique des sciences nous permet de montrer la manière dont les élèves s'approprient les savoirs en jeu, dans le cas particulier des sciences expérimentales, en relation avec l'organisation et des changements d'organisation de l'activité du professeur.

Revenons sur l'analyse de l'activité de mesure du poids. Du point de vue de la didactique des sciences, nous montrons que la nature de la tâche prescrite aux élèves a évolué au cours de ces deux années (\$3.1.2.b). La première année, ils étaient dans la découverte du dynamomètre suivant une démarche empirique. La seconde année, ils étaient dans l'application de la définition du poids, co-construite avec le professeur. Or, du point de vue de la didactique professionnelle, nous analysons que l'organisation de l'activité de l'enseignant est restée la même (figure 4). Nous en déduisons que la nature de la tâche prescrite aux élèves peut changer quand le but de l'activité du professeur ne change pas. En agissant de la sorte, les professeurs atteignent leur but.

Nous avons identifié de nouvelles connaissances professionnelles, de type PCK, qui ont conduit Henri à modifier partiellement sa préparation du cours. Du point de vue de notre modèle, ces modifications se situent sur différents éléments du schème de l'enseignant, suivant le type de boucle de régulation rétroactive qui a eu lieu. D'une part, nous pouvons décrire l'organisation de l'activité des professeurs ainsi que les réorganisations opérées et, d'autre part, nous pouvons distinguer les connaissances nécessaires pour réguler l'activité, des connaissances utiles pour prélever et sélectionner l'information pertinente. Notre analyse du point de vue de la didactique des sciences montre les conséquences de ces réorganisations sur les apprentissages des élèves. La deuxième année, le modèle de la gravitation a été construit par le professeur comme un modèle précur$\operatorname{seur}^{8}$ (Lemeignan \& Weil-Barais, 1993) et a permis la co-construction de la définition du poids, contrairement à la première année. Les élèves ont acquis une connaissance phénoménologique qui élargit le référent empirique au dynamomètre. De fait, il y a eu modélisation et application du modèle selon le 
schéma de Martinand. La première année, ils sont restés dans le registre du référent empirique qui ne leur a pas permis de distinguer le poids de la masse.

Au vu de ces résultats, nous pensons qu'il ne s'agit pas de séparer, à tout prix, le poids et la masse comme l'indiquent les deux enseignants. La construction d'un modèle précurseur, celui de l'interaction, pourrait être intégré comme phénoménologie au niveau du référent empirique afin de décrire le système mécanique étudié en termes : d'objets, d'interaction entre ces objets (agir sur), de propriétés de l'interaction, le tout mis en correspondance avec les événements. Selon Lemeignan et Weil-Barais, il permet de mettre de côté momentanément les difficultés liées à la gestion d'une grandeur vectorielle. Puis, l'application à des situations nouvelles donnerait aux élèves certains automatismes de pensée et la construction de connaissances phénoménologiques afin d'appréhender au mieux le concept de force. Ils pourraient distinguer plus facilement le poids et la masse.

\section{CONCLUSION}

Notre travail nous a conduits à analyser les connaissances mobilisées par les enseignants dans la mise en œuvre de leur préparation, du point de vue de la didactique des sciences et de la didactique professionnelle. En nous appuyant sur des références théoriques et sur une méthodologie construite pour cette recherche, nous avons identifié les connaissances professionnelles des professeurs en articulant le modèle des PCK et le concept de schème.

Notre étude montre que les enseignants mobilisent d'autres types de connaissances que les connaissances disciplinaires académiques : des connaissances sur les élèves, des connaissances sur les programmes, des connaissances sur les stratégies d'enseignement. Ces connaissances dépendent du contenu à enseigner, elles sont des catégories de PCK. Elles s'amalgament (Shulman, 1986) notamment avec des connaissances disciplinaires (SMK), d'un niveau supérieur à celui qui est enseigné, et des connaissances sur le contexte de classe. Toutes ces catégories sont incluses dans les connaissances professionnelles de l'enseignant. Elles lui permettent de rendre le sujet d'étude plus compréhensible pour les élèves (ibid.). Notre analyse à l'aide de la didactique professionnelle permet de distinguer les connaissances nécessaires pour réguler l'activité, des connaissances utiles pour prélever et sélectionner l'information pertinente, dans l'organisation de l'activité du professeur.

La préparation de la classe n'est pas définitive et n'assure pas les enseignants d'atteindre leurs objectifs ; elle évolue à travers des réajustements. Dans cette étude, nous avons fait référence à trois types de régulation : en boucle courte, en boucle longue et en boucle « changement de schème ». Elles montrent que les modifications, et en conséquence l'adaptation de la préparation de l'enseignant se fait à des échelles de temps différentes. Nos résultats indiquent que l'origine d'une boucle de régulation est une nouvelle connaissance de type PCK sur les élèves. La boucle courte implique que le spectre des contenus enseignés est plus large que prévu. C'est une raison pour laquelle un enseignant n'est pas assuré d'apporter une réponse pertinente, à chaque incident critique. La régulation en boucle longue nous permet de montrer comment la capitalisation des connaissances dans l'invariant opératoire et dans l'inférence du schème de l'enseignant, issue de pratiques de classes passées, lui permet de produire une réponse structurée et précise pour les élèves. L'incident critique devient un incident prévu. Son organisation est identique à celle de l'incident critique. La régulation de type « changement de schème » a pour conséquence une réorganisation partielle de l'activité de l'enseignant car un théorème-en-acte n'est plus tenu pour vrai dans l'activité. Pour nous, ce théorème contient une connaissance de type PCK sur les stratégies. Cette boucle de régulation aurait pour origine des PCK qui font système. Elles seraient acquises à différents moments de la séquence et seraient sauvegardées tout au long de la séquence par l'enseignant. Cette réorganisation nécessite de la part de l'enseignant une réécriture partielle de sa préparation.

Le croisement des deux didactiques nous permet de montrer, d'une part, que les régulations de types boucle longue ou boucle " changement de schème " augmentent la participation des élèves à l'élaboration des savoirs en classe. D'autre part, nous observons, dans le processus de modélisation, une projection du référent empirique sur le registre des modèles et une application du modèle, suivant le schéma de Martinand. Les apprentissages sont alors favorisés et l'enseignant peut atteindre son but avec plus de facilités. Ce croisement permet également de montrer que la nature de la tâche prescrite peut évoluer sans que le but de l'enseignant ne change. 
Alain Jameau

Nous l'avons dit, notre approche articule le modèle des PCK et le concept de schème afin d'identifier les connaissances mobilisées par les enseignants. Dans cette étude, nous avons soulevé deux questions théoriques. L'une concerne la pertinence du concept d'invariant opératoire du schème des enseignants et l'autre est à propos du modèle de Magnusson et al. (1999). Nous avons mentionné notre difficulté à identifier des concepts-en-acte dans l'activité des enseignants. Selon Vergnaud, les invariants opératoires échappent à la conscience du sujet. Ils sont aussi très difficiles à expliciter car ils sont intégrés dans l'exécution de l'activité (Coulet, 2010). Les théorèmes-en-acte associés à la notion d'indices ne sont-ils pas suffisants pour comprendre l'activité des enseignants ? Nous rejoignons d'autres résultats de la recherche dans nos difficultés à identifier des PCK sur les stratégies. Qu'est-ce qui est stratégique dans l'action du professeur ? Est-ce le dispositif utilisé indépendamment du contenu ou est-ce le tout?

Nous pensons que notre étude permet d'identifier quelques éléments concernant l'acquisition d'expérience des enseignants. En effet, les incidents critiques sont à l'origine de la construction de nouvelles connaissances, de type PCK sur les élèves. Elles engendrent parfois des boucles de régulation rétroactives de l'activité qui montrent comment ces connaissances participent à l'adaptation de l'enseignement du professeur à la classe, suivant une échelle de temps plus ou moins large. De fait, les mécanismes qui pourraient modéliser, en partie, l'acquisition d'expérience professionnelle sont l'acquisition de nouvelles PCK, la capitalisation des inférences et des invariants opératoires dans les schèmes, la construction de nouveaux schèmes ou de groupements organisés de type incident prévu. Ce qui nous ferait envisager la notion d'expérience à la fois comme un ensemble de schèmes de l'enseignant que nous identifions dans l'action et qui ont été précédemment construits. Mais cette étude demande à être poursuivie notamment en la replaçant par rapport aux travaux sur le développement professionnel des enseignants.

Toutes ces questions montrent le travail qu'il nous reste à accomplir pour mieux comprendre l'activité des enseignants et ses évolutions du point de vue des connaissances professionnelles. Notre approche sous l'angle des deux didactiques apporte des résultats pour la recherche et pour la formation des professeurs qui nous semblent importants de partager avec les deux communautés de didacticiens.

\section{NOTES}

1. Nous désignons ici par préparation l'activité du professeur en amont de la mise en œuvre d'une séance ou d'une séquence. Elle peut donner lieu à la conception et à l'organisation de supports matériels (fiches de préparation...).

2. Nous appelons transcript les tableaux des transcriptions des productions verbales des situations de classe, et des entretiens, associées à quelques descriptions succinctes du chercheur.

3. On a marché sur la Lune, Hergé, Édition Casterman.

4. La distinction entre la masse «inerte » et la masse " grave » n'est pas au programme au collège. Pour eux, la masse désigne la quantité de matière constituant un corps. 5. Les termes «poids» et « l'attraction» sont soulignés par le professeur au tableau.

6. Le poids et la masse, ScienceEVie Junior (157) Octobre 2002.

7. Plus précisément, la fusée tombe à la même vitesse que les héros à l'intérieur.

8. Pour les auteurs, « le qualificatif précurseur associé au mot modèle signifie qu'il s'agit de modèles préparant l'élaboration d'autres modèles. En conséquence, les modèles précurseurs comportent un certains nombres d'éléments caractéristiques des modèles savants vers lesquels ils tendent. Les modèles précurseurs constituent ainsi des constructions didactiques conçues pour aider les élèves à accéder aux modèles savants. Ils sont donc précurseurs au regard du développement cognitif. Ce ne sont pas des précurseurs historiques » (p. 26). 


\section{RÉFÉRENCES}

Abell, K. (2007). Research on science teacher knowledge. Dans K. Abell, \& N. Lederman (dir.), Handbook of research on science education (p. 1105-1150). Mahwah, New Jersey : Lawrence Erlbaum Associates.

Bécu-Robinault, K. (2007). Connaissances mobilisées pour préparer un cours de sciences physiques. Aster, 45, 165-188.

Bisault, J. (2011). Contribution à l'élaboration curriculaire d'une éducation scientifique à l'école primaire : modélisation des moments scolaires à visée scientifique. Mémoire d'habilitation à diriger des recherches. École normale supérieure de Cachan.

Clot, Y., \& Faïta, D. (2000). Genres et styles en analyse du travail. Concepts et Méthodes. Travailler (4), 7-42.

Clot, Y., Faita, D., Fernandez, G., \& Scheller, L. (2001). Entretiens en autoconfrontation croisée : une méthode en clinique de l'activité. Éducation permanente, 146(1), 17-25.

Coulet, J.-C. (2010). Mobilisation et construction de l'expérience dans un modèle de la compétence. Travail et apprentissages, 6, 181-198.

Coulet, J.-C. (2011). Une approche psychologique de la gestion des compétences, au-delà de l'opposition expert/novice. Communication présentée au colloque GESCO, Clermont-Ferrand.

Cross, D. (2010). Action conjointe et connaissances professionnelles de l'enseignant. Éducation \& Didactique, 4(3), 39-60.

Flanagan, J. (1954). The critical incident technique. Psychological bulletin, 51(4), 327-358.

Genzling, J.-C., \& Pierrard, M.-A. (1996). La modélisation, la description, la conceptualisation, l'explication et la prédiction. Dans J.-L. Martinand, \& al, Nouveaux regards sur l'enseignement et l'apprentissage de la modélisation en sciences (p. 46-78). Paris : INRP-Lirest.

Glaser, B., \& Strauss, A. (1967). The discovery of grounded theory. Strategies for qualitative research. Chicago : Aldine.

Grossman, P. (1990). The making of a teacher: Teacher knowledge and teacher education. New York: Teachers College Press.

Gueudet, G., \& Trouche, L. (2010). Des ressources aux documents, travail du professeur et genèses documentaires. Dans G. Gueudet, \& L. Trouche (dir.), Ressources vives. Le travail documentaire des professeurs en mathématiques (p. 57-74). Presses Universitaires de Rennes et INRP.

Jameau, A. (2012). Les connaissances mobilisées par les enseignants dans l'enseignement des sciences. Analyse de l'organisation de l'activité et de ses évolutions (Thèse de doctorat en SHS, Université de Bretagne Occidentale, Brest).

Kermen, I., \& Méheut, M. (2008). Mise en place d'un nouveau programme à propos de l'évolution des systèmes chimiques : impact sur les connaissances professionnelles d'enseignants. Didaskalia, 32, 77-116.
Larcher, C. (2003). Contribution à la table ronde « cadres théoriques autour de la modélisation ». Dans V. Albe, C. Orange, \& L. Simonneaux (dir.), Actes des $3^{\text {es }}$ rencontres scientifiques de l'ARDIST. Recherches en didactique (p. 305-308). Toulouse : ENFA.

Lemeignan, G., \& Weil-Barais, A. (1993). Construire des concepts en physique. Paris: Hachette éducation.

Leontiev, A. (1972). Le développement du psychisme. Paris : Éditions sociales.

Leplat, J. (2004). Lanalyse psychologique du travail. Revue Européenne de Psychologie Appliquée, 2(54), 101-108.

Leplat, J. (2006). Les contextes de formation. Éducation Permanente, 166, 29-48.

Magnusson, S., Krajcik, J., \& Borko, H. (1999). Nature, sources and development of pedagogical content knowledge for science teaching. Dans J. Gess-Newsome, \& N. Lederman (dir.), Examining pedagogical content knowledge: The construct and its implications for science education (p. 95-132). Boston : Kluwer.

Martinand, J.-L. (1996). Introduction à la modélisation. Actes du séminaire des didactiques des disciplines technologiques, 1-12. Cachan (1994-1995) : Association Tour 123.

Martinand, J.-L. (2000). Rapport au savoir et modélisation en sciences. Dans A. Chabchoub (dir.), Rapports aux savoirs et apprentissage des sciences. Actes du $5^{\mathrm{e}}$ colloque international de didactique et d'épistémologie des sciences, tome 1 (p. 123-135). Tunis.

Morge, L. (2003). Connaissances professionnelles locales: cas d'une séance sur le modèle particulaire. Didaskalia, 23, 101-131.

Munoz, G. (2006). Filiations et ruptures en didactique professionnelle. Éducation Permanente (166), 87-103.

Pastré, P. (1997). Didactique professionnelle et développement. Psychologie française, 42(1), 89-100.

Pastré, P. (1999). La conceptualisation dans l'action : bilan et nouvelles perspectives. Éducation Permanente, 139, 13-35.

Pastré, P. (2008). Introduction. Dans P. Pastré, \& Y. Lenoir, Didactique professionnelle et didactqiue disciplinaire en débat (p. 13-17). Toulouse : Octares.

Pastré, P. (2011). La didactique professionnelle. Paris : PUF.

Pastré, P., Vergnaud, G., \& Mayen, P. (2006). La didactique professionnelle. Revue française de pédagogie, 154, 145198.

Piaget, J. (1974). Réussir et comprendre. Paris : PUF.

Power, M. (2008). Le concepteur pédagogique réflexif : un journal de bord. Athabasca, AB : Athabasca University Press.

Rabardel, P. (2007). Principes pour la construction d'une didactique professionnelle. Dans Merri (dir.), Activité humaine et conceptualisation. Questions à Gérard Vergnaud (p. 87-90). Toulouse: Presses universitaires du Mirail.

Samurçay, R., \& Rabardel, P. (2004). Modèles pour l'analyse de l'activité et des compétences, propositions. Dans R. Samurçay, \& P. Pastré (dir.), Recherches en didactique professionnelle (p. 16-180). Toulouse : Octarès. 
Sanchez, E. (2008). Quelles relations entre modélisation et investigation scientifique dans l'enseignement des sciences de la Terre ? Éducation E Didactique, 2(2), 97122.

Sensevy, G., \& Amade-Escot, C. (2007). Une présentation de "Those who understand knowledge growth in teaching". Éducation E Didactique, 1(1), 95-96.

Sensevy, G., \& Mercier, A. (2007). Agir ensemble. L'action conjointe du professeur et des élèves dans le système didactique. Rennes: PUR.

Shulman, L. S. (1986). Those who understand : Knowledge growth in teaching. Educational researcher, 15(2), 4-14.

Shulman, L. S. (1987). Knowledge and teaching : Foundations of the new reform. Educational Review, 57(1), $1-22$.

Vergnaud, G. (1990). La théorie des Champs Conceptuels. Recherches en didactiques des mathématiques, $10(2,3)$, 133-170.

Vergnaud, G. (1996). Au fond de l'action, la conceptualisation. Dans J. BARDIER (dir.), Savoirs didactiques et savoirs d'action. Paris : PUF.

Vergnaud, G. (2001). Piaget visité par la didactique. Intellectica, 33, 107-123.

Vergnaud, G. (2008). De la didactique des disciplines à la didactique professionnelle, il n'y a qu'un pas. Revue Travail et Apprentissages, 1, 51-57.

Vinatier, I. (2009). Pour une didactique professionnelle de l'enseignement. Rennes: PUR.

Walliser, B. (1977). Systèmes et modèles. Introduction critique à l'analyse de systèmes. Paris : Le Seuil. 